\title{
EN EL LUGAR DEL OTRO: POSIBILIDADES ÉTICO- EPISTEMOLÓGICAS PARA LAS CIENCIAS HUMANAS
}

\section{IN PLACE OF OTHER: ETHICAL AND EPISTEMOLOGICAL POSSIBILITIES TO THE HUMAN SCIENCES}

\author{
Juan Carlos Aguirre ${ }^{1}$, Luis Guillermo Jaramillo \\ ${ }^{1}$ Doctorante en Filosofía, Universidad del Cauca, Ciudadela Campestre La Rioja, Casa i-7 Popayán, Cauca, Colombia, \\ jcaguirre@unicauca.edu.co; ${ }^{2}$ Doctor en Ciencias Humanas y Sociales-Educación, Universidad del Cauca, Ciudadela \\ Campestre La Rioja, Casa i-2 Popayán, Cauca, Colombia, ljaramillo@unicauca.edu.co
}

Rev. U.D.C.A Act. \& Div. Cient. 16(2): 535-542, Julio- Diciembre, 2013

\section{RESUMEN}

Este artículo, se propone confrontar la propuesta ética del filósofo Emmanuel Levinas con algunas recepciones que se hacen de ella, en el contexto de las ciencias humanas, especialmente, en la psicología, con el fin de determinar sus aportes de orden epistemológico. El trabajo, se centrará en el concepto substitución (para-el-otro). En primer lugar, se expondrá lo que el autor entiende por substitución; posteriormente, se analizará el enfoque de la Psicología para el Otro, expuesta por George Kunz. Finalmente, se revisará de modo crítico tal enfoque y se describirá los aportes que la ética de Levinas podría ofrecer para la fundamentación epistemológica de las ciencias humanas.

Palabras clave: Otro, psicología fenomenológica, respuesta, ciencias humanas y sociales, substitución.

\section{SUMMARY}

This article is aimed to confront the ethical proposal of the philosopher Emmanuel Levinas with some conceptions about it in the framework of Human Sciences, especially in the Psychology, in order to determine their epistemological contributions. The dissertation is focused in the concept of substitution (for-the-other). Firstly, it shows what the author understands as substitution; secondly, it analyzes the approach of the Psychology for the other, exposed by George Kunz. Finally, it reviews critically that approach and it describes contributions that Levinas' Ethic would offer to the epistemological foundation of Human Sciences.

Key words: Other, phenomenological psychology, response, social and human sciences, substitution.

\section{INTRODUCCIÓN}

La relación entre Levinas y las ciencias humanas y sociales ha sido poco explorada, pese a que en muchos trabajos, de quienes producen conocimiento en tales ciencias, se nota la influencia de sus tesis. Véase, a modo de ejemplo, los siguientes trabajos: Bauman (1996) retoma las categorías levinasianas, tanto de "extranjero" como de "proximidad", para consolidar su análisis de la ambivalencia, que se antepone a la modernidad; Le Breton (1999), en su texto Antropología del dolor, recurre a Levinas para respaldar su tesis de la incomunicabilidad del dolor, haciendo eco de los profundos análisis de Levinas (1993); finalmente, Mélich con su texto Ética de la Compasión (2010).

Además de la abigarrada escritura de Levinas, se suman las múltiples interpretaciones que de él se han hecho, al punto de tener que parafrasear a Bernasconi (2012), afirmando que habrá que hacer una crítica filosófica levinasiana de la filosofía levinasiana; sin embargo, una de las dificultades de la filosofía levinasiana ha sido que, para algunos, esta nada tiene que ver con cuestiones epistemológicas, toda vez que en la instauración de la ética, como filosofía primera, Levinas ha hecho una demolición radical de todo presupuesto que esté amparado o conduzca a situaciones que atañen con la epistemología; no obstante, en algunos trabajos hemos controvertido este prejuicio, intentando encontrar lazos que se puedan tender entre la filosofía de Levinas y la epistemología de las ciencias humanas y sociales (Aguirre \& Jaramillo, 2006; Jaramillo \& Aguirre, 2010; 2012); lo anterior, tratando de conservar la propuesta levinasiana y viendo en ella los modos de considerar una ciencia humana y social, acorde con las exigencias que superen el positivismo, sin tener por ello que renunciar a la racionalidad (Giere, 2006; Mélich, 2010). 
Un buen número de aproximaciones a Levinas, asumidas desde las ciencias humanas y sociales, se realiza de manera metódica, extrayendo de la obra del filósofo aspectos nucleares, que fundamentan algún nodo particular de la reflexión (Dale, 2012; Idareta, 2012); sin embargo, pensadores agrupados alrededor de lo que Garza \& Landrum (2010) denominan Escuela de Seattle, han ido consolidando un programa de investigación -fundamentado en la obra de Levinas-, donde se propone una Psicología para-el-Otro, liderado por el psicólogo y fenomenólogo, Kunz.

Ahora bien, la expresión para-el-Otro remite a un concepto fundamental de la segunda gran obra de Levinas: Autrement qu'être ou au-dela de l'essence (1974/2004), a saber, el concepto de substitución (Substitution), del cual, Levinas afirma: "toda acusación y persecución, así como todo elogio, recompensa o castigo interpersonales, suponen la subjetividad del yo (Moi), la substitución, la posibilidad de ponerse en el lugar del Otro que se refiere a la trasferencia del «por el Otro» al "para el Otro»" (2004); según Waldenfels (2011), substitución se entiende como "en el lugar del Otro".

\section{MATERIALES Y MÉTODOS}

Con base en las anteriores consideraciones, el presente artículo de reflexión, se propone confrontar la propuesta ética de Levinas con algunas recepciones que se hacen de ella, en el contexto de las ciencias humanas y sociales, especialmente, en la psicología propuesta por Kunz y sus colegas de la Escuela de Seattle. El objetivo es determinar qué elementos de la obra de Levinas sustentan epistemológicamente la propuesta, a la vez, que revisar críticamente tales elementos, para tratar de extender sus posibilidades a otras disciplinas humanas y sociales. Para lograr este propósito, se comenzará presentando, esquemáticamente, la tesis levinasiana de la substitución, puesto que es ella la que inspira el enfoque psicológico de Kunz. Posteriormente, se expondrán las tesis fundamentales de la propuesta Psicología-parael-Otro tal y como Kunz la presenta en algunos de sus textos más relevantes. Finalmente, se plantearán algunas consideraciones críticas al respecto y se indicarán los modos cómo se puede extender el caso de Kunz a otras ciencias sociales.

\section{RESULTADOS Y DISCUSIÓN}

Substitución: la posibilidad de ponerse en el lugar del otro: Para algunos especialistas de la obra de Levinas, entre la primera gran obra del autor, Totalité et infini (1961/1990) y la segunda, Autrement qu'être ou au-dela de l'essence (1974/2004), se da una especie de transición; al respecto señala Bettina Bergo (2011): "en el corazón del trabajo de 1961 estaba el Rostro como expresión y voz enseñanza. El ensayo de 1974 presenta un encuentro menos fenomenalista, menos iluminado, llamado 'substitución'”. Es así, que el concepto se vuelve preponderante a la hora de comprender la obra de Levinas como un todo. El propio filósofo así lo reconoce, cuando señala que la consideración acerca de la substitución (que en el libro de 1974 ocupa el capítulo IV), "fue el germen de Autrement qu'être ou au-dela de l'essence" (Levinas, 2004). Curiosamente, el concepto parece tornarse en ocasiones enigmático, al punto que algunos se preguntan insistentemente cuál es la pregunta que la palabra substitución pretende responder (Bernasconi, 2004). En tanto, no es asunto de este escrito dar respuesta a la pregunta que se hace Bernasconi, sobre todo porque exigiría una aproximación exegética de concepto (trabajo hecho en Aguirre, 2012), baste con presentar una sintética definición de lo que es la substitución, con el fin de exponer, posteriormente, sus aristas conceptuales.

Según Calin \& Sebbah (2002), la substitución se puede definir como: "ponerse en el lugar del otro', estar a cargo, llevar los sufrimientos y las culpas del otro, y por tanto, ser acusado y expiar unos pecados que yo (je) no he cometido, que no comenzaron en un mí (moi). Aquí no hay lugar bajo

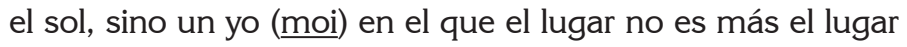
donde él se posa, más bien, el 'no-lugar' (AE 148) donde se soportan todos los sufrimientos y las culpas de los otros. El

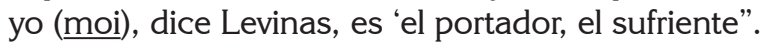

De esta definición, se pueden extraer varios elementos. En primer lugar, substitución indica "ponerse en el lugar del otro"; es decir, "conduce a una relación tópica: "A aparece en lugar de B”. Pero si nos detenemos aquí, nos enredaríamos en la perspectiva del observador neutral, describiendo relaciones dadas sin involucrarse con alguna de ellas" (Waldenfels, 2011). De acuerdo con esto, la substitución levinasiana, entendida como "en el lugar del otro", tendrá que superar el carácter de "punto dado en un espacio homogéneo" (ibíd). No se trata, entonces, de un ejercicio en el que un individuo trata de ubicarse, real o imaginariamente, en un lugar o espacio desde el cual, comprende aquello que está, o bien en el campo perceptivo del otro (espacio físico), o bien en una situación mental (situación psicológica); tampoco se trata de substitución, en el sentido de que alguien deje de ser para volverse lo ajeno a él, pues como lo ha señalado la tradición fenomenológica, en la que Levinas se inscribe, "defender que yo solo tendría una vivencia real del otro si experimento sus sentimientos o pensamientos de la misma manera como él lo hace, es un sinsentido. Esto implica que solo vivenciaría al otro si lo hiciera de la misma forma que me experimento a mí mismo; conduciría por tanto a una abolición de la diferencia entre el yo y el otro" (Husserl, 1986; Gallagher \& Zahavi, 2008; Zahavi \& Overgaard, 2013). En consecuencia, substitución como "en el lugar del otro", debe tener otra connotación en la obra de Levinas. 
Siguiendo la definición de Calin y Sebbah, se puede ver, en segundo lugar, la introducción de una serie de conceptos extraños a los discursos de la filosofía, en general y la epistemología, en particular. Se habla de "sufrimientos", "culpas", "ser acusado", "expiar unos pecados"; conceptos más cercanos, probablemente, a las tradiciones teológicas; sin embargo, se considera que el cambio de vocabulario no conduce, necesariamente, a una adopción del discurso religioso; de lo que se trata más bien, es de llamar la atención sobre los nuevos modos de enunciación de lo humano; modos que bajo ninguna circunstancia pueden agotarse en "lo dicho" (Levinas, 2004).

Los modos típicos de "lo dicho" confrontan algunas ideas que se han entronizado en lo que se ha dado en llamar Pensamiento Moderno, específicamente, la idea de la primacía del sujeto y la idea de la libertad individual. La comprensión del concepto levinasiano de substitución tendrá que des-sedimentar ambas ideas tan caras a nuestra tradición de pensamiento.

Frente a la primera idea, Levinas sostiene que la relación originaria no se da, como se ha creído hasta el presente, como una aparente salida del yo que reflexiona sobre sí mismo; o mejor, como la salida del yo con la única pretensión de volver a sí mismo (en este punto, Levinas (1967) recurre a la figura de Ulises). De modo contrario, la relación más originaria (anárquica) es postulada por Levinas, al margen de la conciencia que trata de relacionarlo todo consigo misma; lo anárquico, esto es, lo previo a toda tematización, se sitúa en el terreno de la sensibilidad-proximidad. En este punto sostiene Levinas (2004): la proximidad apareció como la relación con el Otro que no puede resolverse en 'imágenes' ni exponerse como tema mediante aquello que no es desmesurado con relación a la arjé de la tematización, sino que es inconmensurable mediante aquello que no mantiene la identidad del logos kerigmático poniendo en entredicho todo esquematismo.

Con relación a la segunda idea -que tiene que ver con la libertad individual-: en la medida en que la estructura original de subjetividad no es primeramente la identidad (el sujeto que sale de sí para verse reflejado a sí mismo o el sujeto que todo lo mide de acuerdo con las intenciones de su conciencia), es la sensibilidad-proximidad quien prefigura todo desarrollo posterior, la libertad individual no se instaura por sí misma sino que se pone en cuestión por la presencia del otro. Esta puesta en cuestión saca al yo de su comodidad y exige una respuesta impostergable e irremplazable: "Al no poder remitirse la relación de proximidad a cualquiera de los modos propios de la distancia o de la continuidad geométrica, ni tampoco a la simple "representación" del prójimo, tal relación es ya asignación de una extrema urgencia, obligación anacrónicamente anterior a todo acto de compromiso. Anterioridad "más antigua" que el a priori (...) Hemos llamado obsesión a esta relación con la exterioridad "anterior" al acto que la abriría, relación que no es precisamente un acto, que no es tematización" (Levinas, 2004).

La substitución, lejos de tornarse un mero intercambio de espacios o roles entre el yo y el otro; lejos de tomar la vocería de otro que no tenga posibilidad de hablar, se vuelve la actitud de un yo portador y sufriente, de un yo que no puede eludir su responsabilidad, de un yo "rehén" (Levinas, 2004). Esta substitución, en sentido originario, implica que "Comienzo desde el lugar del Otro. Comienzo desde donde no puedo estar y desde donde todo lo que me llama la atención: la mirada del Otro, la palabra del Otro o el gesto del Otro, tiene su origen. En cierta medida, soy el Otro para quien substituyo; los Otros no son duplicados de mí, sino dobles que encontramos desde una distancia por fuera de nuestro alcance" (Waldenfels, 2011). Ahora bien, según el presente concepto de substitución: cंqué fundamentos teóricos permiten hacer funcionales las tesis de Levinas en el terreno de la psicología?

La substitución y la psicología para el otro: El psicólogo George Kunz (1998) propone de modo articulado el enfoque de la Psicología para el Otro, el cual, pretende erigirse como un "paradigma alternativo para la psicología". Por tanto, se intentará mencionar en qué consiste tal enfoque para luego evaluar, en un apartado final, si se constituye o no en un paradigma alternativo, así como establecer posibilidades concretas para la investigación en ciencias humanas.

En su libro: The paradox of power and weakness (1998), Kunz se propone presentar la visión de lo que denomina "altruismo radical", que se levanta como paradigma alternativo a los paradigmas típicos de la psicología moderna, encerrados en el auto-interés. Desde el título del libro, se puede percibir un intento por identificar el concepto de poder con la psicología moderna; se comprende, por tanto, que el paradigma alternativo tendrá que ser abordado desde el concepto de debilidad (weakness), el cual, a partir de esta lente, lee la obra de Emmanuel Levinas. De otra parte, es importante mencionar, de entrada, que lo interesante del trabajo de Kunz (2006) consiste en su pretensión de no salirse del marco de cientificidad; por el contrario, Kunz considera que la cientificidad está en bancarrota y es preciso recomponerla con concepciones más radicales de ciencia, consideración que coincide con el padre de la fenomenología Edmund Husserl (1976 y 2009).

Ahora bien, sin mencionarlo por ningún lado, se puede sospechar que Kunz asume la imagen de ciencia y de cambio científico según el esquema propuesto por Kuhn (1996), especialmente, en sus componentes de "paradigma" 
de la ciencia normal, "anomalías" y paradigma emergente u alternativo (literalmente Kuhn habla de un "nuevo paradigma que emerge"); por tanto, el nuevo paradigma, según Kunz, tendría pretensiones de tornarse una revolución en los estudios psicológicos. A continuación, se exponen las tesis de este autor, siguiendo este esquema: Kunz (1998) comienza postulando que, actualmente, se percibe un ambiente de "nihilismo cínico", formulado por la postmodernidad, a partir de las promesas incumplidas por el proyecto moderno. Su análisis lo lleva a sintetizar las notas características de la modernidad mostrando sus promesas, tanto en el ámbito de lo meramente físico como de lo humano, para luego señalar que, tales promesas, se tornaron desilusiones.

De acuerdo con esto, Kunz afirma que el espectro de la modernidad está constituido por lo siguiente: En primer lugar, "la ilustración cognitiva y la liberación de la ignorancia": tanto la ciencia natural como la humana fueron acumulando conocimiento reflejado en la producción de métodos, teorías, hechos; así como en sus resultados: "las verdades"; sin embargo, aunque en la ciencia natural seguimos siendo ingenuos acerca de gran parte de su acervo teórico, en las ciencias humanas y sociales parece que el conocimiento se ha ido popularizando entre el gran público: "nos consideramos a nosotros mismos perceptivos de las intenciones y motivos de los otros y, en especial, de nosotros mismos. Este conocimiento moderno nos da confianza para juzgar a las personas en la cotidianidad, planear cursos de acción y disfrutar nuestra sofisticada auto-confianza al imaginar los significados de las personas" (Kunz, 1998).

En segundo lugar, Kunz habla de la "eficiencia conductual", la cual, se refleja en el ámbito de lo físico mediante las acciones de las máquinas, que han permitido ampliar el conocimiento de la naturaleza de las cosas; en el ámbito de lo humano, por su parte, "la eficiencia en la administración de las acciones individuales y sociales nos permite aligerar nuestros sistemas organizativos para cumplir eficientemente el trabajo productivo". Como consecuencia de esto, las personas se pueden entrenar para que sean trabajadores efectivos en el desarrollo de tareas complejas, lo que conduce a que los contratiempos se traduzcan como errores evitables, si se aplican las técnicas apropiadas. En el terreno de la psicología y la administración, "la modernidad ha reducido, aparentemente, la probabilidad de fracaso". Finalmente, y en tercer lugar, "el disfrute afectivo de los productos disponibles al consumidor ha contribuido a la creación de un estilo de vida confortable para la mayoría de los ciudadanos" (Kunz, 1998).

Bien se sabe que estas características han ido perdiendo vigencia en nuestro contexto contemporáneo: se ha desacreditado la vieja imagen de la ciencia, se han puesto en cuestión, tanto el conductismo en psicología como sus versiones en administración, educación y demás; por último, hemos llegado al punto que el pretendido dominio de la naturaleza para satisfacción de las mayorías no fue más que un sofisma que excluye pueblos y regiones geográficas y es inmisericorde con los recursos naturales. Ante esto, el discurso de la postmodernidad, expuesto de diversas maneras por autores, como Lyotard (1979) o Latour \& Woolgar (1979), parece presentar salidas; sin embargo, tales salidas no han conducido sino a un "nihilismo cínico" (Haack, 2007; Sokal, 2010), cuyo centro está constituido por una radicalización del yo, una egología en la que, incluso, el interés por el otro está mediado por procesos de autosatisfacción.

En lo que respecta a la psicología, Kunz advierte que ella ha contribuido a la consolidación de este "nihilismo cínico", pues "la psicología hoy, ciertamente aquella clase que más impacta la cultura popular, atiende exclusivamente al estudio del ego. La psique de la psicología moderna es el ego que se establece a sí mismo en el centro de la personalidad individual, construyendo su propia identidad, manipulando su ambiente para saciar sus necesidades y disfrutar el gozo que produce el satisfacer esas necesidades" (Kunz, 1998). En este sentido, la psicología ha perdido de vista la capacidad que tiene el ser humano de trascender sus necesidades para encontrar un deseo más profundo, reduciéndose a mera contemplación del yo.

Esta situación, denuncia Kunz, no es esencial a la psicología, es decir, esta no debe reducir su campo de investigación al yo y sus procesos; es por esto que Kunz se da a la tarea de definir el término "psicología" desde sus orígenes, mostrando cómo la raíz griega psukhe remite originalmente a aliento (breath), inspirar. Para Kunz, "esta antigua psukhe no engendra el sí mismo ni dirige sus esfuerzos hacia el sí mismo [...]. La psukhe era un don de los otros" (1998).

La contraposición, que no es meramente sintáctica, se da entre la psicología (denominada por Kunz como egología) y la psukhología. Alrededor de estos conceptos, se establece la diferenciación entre el poder y la debilidad: el poder es la preocupación por la identidad, el empoderamiento de esta identidad, la evitación de la debilidad, el encuentro del potencial individual y la preocupación por el auto-desarrollo; la debilidad es "el estudio del yo teniendo su identidad inspirada por los otros, animada por los otros, empoderada por los otros" (Kunz, 1998). La pretensión no es solamente confrontar dos modos de considerar la psicología, sino plantear la psukhología (un don de los otros) como un paradigma emergente frente al paradigma dominante (egología); paradigma que conduciría a la redefinición de la noción de psicología. Recientemente Kunz (2012) sostiene: "La psique y sus significados han sido olvidados pues no encuadraron bien con el enfoque y el método modelado 
después [del método] de las ciencias naturales. Este enfoque fenomenológico (la Psicología para el otro) es un modo de retornarlos a la psicología".

Antes de explorar el aporte del concepto levinasiano de substitución a la psukhología propuesta por Kunz, se debe decir que tal enfoque no nace de una disyuntiva teórica, sino que parte de las propias anomalías (llamadas por él como "paradojas") que encontró Kunz al desarrollar su práctica como psicólogo. He aquí su propia descripción: "Mientras confirmo nuevamente mi argumento que el mundo no está dividido entre buenos y malos, puedo distinguir adecuadamente entre una egología y una psukhología reconociendo la verdad obvia que el poderoso posee y disfruta su poder, mientras que el débil sufre su debilidad. Notamos, sin embargo, que las personas poderosas sabotean frecuentemente su propio poder, y la debilidad del débil tiene un poder sobre nosotros. Ni el poderoso ni el débil disputan la verdad del poder del poderoso. Algunas personas poderosas pueden estar inconformes con su poder, y algunos débiles muy confortables en su debilidad. Pero el hecho que el poder es poderoso y la debilidad es débil es obvia para todos: el poder empodera, respalda, perpetúa, libera de responsabilidades; la debilidad, sin embargo, debilita, oprime, limita, incluso mata a quien la sufre" (Kunz, 1998).

De las afirmaciones anteriores, se puede sostener que existe una tesis irrefutable acerca del poder y la debilidad; no obstante, hay casos en los que los polos en discusión se tienden a confundir, emborronándose sus límites hasta tornarse auténticas paradojas. Igualmente, a partir de estas dos categorías (poder y debilidad), se puede leer la historia del pensamiento: están quienes defienden las visiones de poder y están quienes rechazan tales pretensiones e intentan reivindicar la figura del débil.

La cuestión es que la psukhología no se ubica en ninguno de estos polos, que bien podrían tener cabida en la lógica del paradigma dominante, sino que trata de afrontar las anomalías (paradojas) por fuera de tal lógica, es decir, toma de frente aquellas relaciones entre el poder y la debilidad, que son leídas como ilógicas (Kunz habla de oxímoron, ampliando su caracterización como "enigmáticas" "ambiguas", "irónicas"), a la luz de la psicología moderna. La formulación de la paradoja que desnuda la lógica de la psicología moderna es la siguiente: "en la base de las luchas individuales y sociales de la debilidad contra el poder y del poder contra la debilidad, encontramos estas paradojas no naturales: el poder nutre su propia debilidad y la debilidad posee su propia fuente de poder en esa debilidad" (Kunz, 1998).

Parafraseando a Kuhn (1996), las anomalías no tienen respuesta efectiva a la luz del paradigma dominante, es por ello que la mayoría de los científicos tienden o a trivializarlas o a dejarlas a un lado. En el caso de Kunz, estas anomalías atraen cada vez más su atención y la de otros investigadores (agrupados alrededor de la Universidad de Seattle), dando origen a una comunidad, que se va zafando de lo hasta ahora asumido como paradigmático en psicología, toda vez que ninguna de las respuestas que se ofrecen, puede satisfacer los requerimientos de las paradojas sugeridas. Como consecuencia, la nueva comunidad tendrá que articular el paradigma emergente a partir de nuevas respuestas a las anomalías encontradas.

Ahora bien, ¿cuáles de estas ideas tienen respaldo en la tesis levinasiana de substitución? En primer lugar, si bien el paradigma propuesto por Kunz se denomina Psicología para el otro, a partir del concepto de psukhología, este para-elotro adopta un carácter diferenciador del mero estar en el lugar del otro. En este punto, la coincidencia con Levinas es sorprendente, pues un para-el-otro en el que la relación se dé en términos de conocimiento ("entiendo lo que te pasa") o egoísmo ("al entender tus motivos quedo tranquilo con mi conciencia”), no sería sino una mera manifestación de la totalidad (Levinas, 1990); en palabras de Kunz, no sería más que un intento por disolver las paradojas que la interacción encierra.

En segundo lugar, connatural a la substitución se halla el descentramiento del ego (psique), lo que no se debe entender como una aniquilación. Para Kunz (1998), lo mismo que para Levinas, "el llamado a la responsabilidad por los otros, define la característica fundamental de la psique humana"; antes que una filosofía del sujeto, está la filosofía de la intersubjetividad o mejor, para salirse del marco estrictamente fenomenológico, una filosofía de la otredad en la que la primacía del otro suspenda la actividad del yo - he aquí los conceptos levinasianos de "libertad cuestionada" (Levinas, 1990) y "pasividad” (Levinas, 2004)_, primacía sin origen en el yo (que en Levinas es "anárquica"), sin libertad del yo para tomar posición. En este punto, Kunz (2007) propone un modo de analizar la psique por fuera de los estudios tradicionales de la psicología moderna.

Finalmente, la substitución o psicología para el otro, adopta claramente un enfoque responsivo, la responsabilidad es su seña específica: "no elegimos responder. Elegimos la manera en la que respondemos. Alejarse es una respuesta. Cada tipo de respuesta es una respuesta. Ninguno elige el llamado, y ninguno elige no responder. Ninguno responde con ninguna respuesta" (Kunz, 1998).

Lo valioso de la propuesta de Kunz es que se encuentra en consonancia con el esquema kuhniano de ciencia, en condiciones como: 1) sirve para estructurar una comunidad 
científica, es decir, "gobierna no un número de cuestiones, sino un grupo de practicantes" (Kuhn, 1996); 2) señala la constelación de compromisos con el grupo y, 3) indica los ejemplos compartidos. En este punto, es necesario recalcar que la psicología para el otro ha ido logrando estatus de paradigma emergente o alternativo, aunque no está establecido sino en competencia con el paradigma dominante, pues teóricamente comparte unas tesis. Varios investigadores asumen estas tesis así como el compromiso por desplegarlas en casos que permitan su aplicación en ejemplos concretos. Señal de ello son las contribuciones a las diferentes versiones del seminario: "Psicología para el otro", entre las que se destacan los siguientes títulos: "Levinas en la práctica: tratamiento de la salud mental en una clínica basada en la comunidad" (Flaxer, 2011), "El gueto interno: cultura y memoria" (Macdonald et al. 2011), "Inclusión: actitudes de los terapeutas clínicos hacia la otredad" (Orange, 2011), "La psique despierta: trauma, violencia y el otro" (Goodman \& Becker, 2009).

En síntesis, la psicología para el otro es un caso tangible de los modos como algunas tesis filosóficas, en este caso, las propuestas por Levinas, se imbrican en desarrollos de las ciencias humanas y sociales, bien sea para fundamentarlas teóricamente o para sugerir nuevas rutas metodológicas o prácticas (terapéuticas en la mayoría de los ejemplos relatados). Seguidos por el ejemplo de Kunz, se propone — como conclusión-, algunas posibilidades de las tesis de Levinas para las ciencias humanas y sociales.

Rutas ético-epistemológicas que deja abiertas Levinas para una investigación en ciencias humanas y sociales: Hay un más allá del instinto y un más acá de la razón, una sensibilidad que me hace responsable, que media, porque encuentro un alguien que quiere decir algo, pero sobre todo, un alguien que quiere escuchar. La denuncia que hace Kunz de la modernidad frente a la ilustración cognitiva y su apuesta por la liberación de la ignorancia, no solamente llevó al enfrascamiento de la psicología como ciencia humana sino a las ciencias sociales en general. Kunz coincide con Levinas respecto a la in-visibilización que sufre el hombre como consecuencia de los procedimientos de idealización ejercidos por las ciencias, pues en la medida que el hombre investiga lo humano como "objeto" de estudio en un proceso social, se investiga a sí mismo.

Desde la modernidad, la experticia del método científico requiere de un investigador que se mueva en medio de una neutralidad valorativa respecto al fenómeno social observado; esto pone en crisis, no solamente el fenómeno mismo, sino al sujeto-experto que lo estudia. Crisis que se complejiza, aún más, en disciplinas — como la psicología—, que intentan comprender la mente del otro bajo una mirada dual y racional, lo cual, deja por fuera componentes que la misma razón no puede in-corporar (poner un cuerpo) por la limitación que el orden lógico-racional le exige. En este sentido, Levinas denuncia el desvarío mental de un hombre que sale de sí mismo - con un fin comprensivohacia otro, en cuyo intento este es negado y borrado en su alteridad; movimiento mental del investigador que hace del extraño algo propio y de su excedente un mismo; así, como sujeto pensante-investigador, retorna a sí mismo gracias al sistema teórico y temático que encapsula todo origen, todo comienzo y todo nacimiento del otro a su mismidad. No en vano, Levinas llamaría la atención a las ciencias sociales argumentado que "la humanidad de lo humano -la verdadera vida- está ausente" (Levinas, 1991).

La formación en investigación, específicamente en investigación cualitativa, ha venido dando un viraje hacia una formación con sentido ético, bajo la premisa de que es necesario e imprescindible romper las estructuras de un pensamiento que obtura el sentido-en-cuanto-vivido de aquellos que se resisten a ser tipificados como ausentes o anónimos flotantes para las ciencias sociales: "agregados con el rostro y el perfil otorgado por la lógica universalista de una institución particular" (Díaz de Rada, 2008).

He ahí la pertinencia de la substitución para la investigación en ciencias sociales, pues esta, en ningún caso, remite a intercambio de espacios y de manejo de roles entre investigador e investigados; usualmente, el investigador es quien cuenta con los "poderes" necesarios para comprender y pre-comprender al otro. Desde la substitución todo "comienzo es desde el lugar del Otro" (Levinas, 2004). En este sentido, el increpado a comprender-se respecto a su acercamiento al otro es el investigador y no el sujeto de investigación; de este modo, el investigador debe re-traerse de su comprensión omnisciente con el fin de mirarse a sí mismo antes de mirar al otro; será su reflexividad frente al otro un camino para asumir al investigado como alguien que excede todo conocimiento acerca de él.

No se trata, entonces, de ponerse en "los zapatos del otro", pues su sentido de caminar implica un calzado que excede la apariencia de los zapatos, para colocarlo, más bien, en los pies de aquellos que han transitado por caminos que han desgastado cantidad de calzados: "quizás la expresión, "estar en los zapatos del otro" (to be in someone else's shoes), sea también un residuo que mantiene poca relación con el ejercicio efectivo de intentar situarse en la posición de otro y aproximarse a su dolor. En todo caso, la expresión de hacer empatía, de ponerse en su lugar, no alude al sombrero, el calzón o la camisa sino precisamente al zapato y eso quizás se explique por su imbricación con lo singular del individuo" (Schindel, 2013). Rapport no significa empatía como comúnmente se expone en los textos de métodos y técnicas en investigación social. Estar frente al otro es implicarse con 
su sufrimiento y su dolor, substitución de mi yo por el yo del otro al hacernos responsables del él, sin méritos. Kunz y Levinas plantean el entierro de nuestros egos en las ciencias sociales, pues estos resultan ser el obstáculo más feroz frente a la relación infinita con el Otro.

Agradecimientos: Este trabajo es fruto de las discusiones adelantadas al interior del grupo de investigación Fenomenología y Ciencia, inscrito en la Vicerrectoría de Investigaciones de la Universidad del Cauca y reconocido por Colciencias. Se da expresamente crédito y mención a la Universidad del Cauca, por brindar los espacios y tiempos necesarios para madurar las reflexiones aquí consignadas. Conflicto de intereses: El manuscrito fue preparado y revisado con la participación de todos los autores, quienes declaramos que no existe ningún conflicto de intereses, que ponga en riesgo la validez de los resultados esperados.

\section{BIBLIOGRAFÍA}

1. AGUIRRE, J. 2012. El uno-para-el-otro o la superación ética de la ontología. Escritos. 20(44):69-82.

2. AGUIRRE, J.C.; JARAMILLO, L.G. 2006. El otro en Levinas: una salida a la encrucijada sujeto-objeto y su pertinencia en las ciencias sociales. Rev. Latinoam. Cienc. Soc., Niñez y Juventud. 2(4):47-71.

3. BAUMAN, Z. 1996. Modernidad y Ambivalencia. En: Beriain, J. (comp). Las consecuencias perversas de la modernidad. Barcelona: Anthropos, p.73-119.

4. BERGO, B. 2011. The Face in Levinas; towards a phenomenology of substitution. Angelaki. J. Theoretic. Humanities. 16:17-39.

5. BERNASCONI, R. 2004. What is the question to which 'substitution' is the answer? En: Critchley, S.; Bernasconi, R. (eds) The Cambridge Companion to Levinas. Cambridge U. Press. p.234-251.

6. BERNASCONI, R. 2012. Levinas's Ethical Critique of Levinasian Ethics. En: Davidson, S.; Perpich, D. (eds). Totality and Infinity at 50. Pittsburgh: Duquesne University Press. p.253-269.

7. CALIN, R.; SEBBAH, F. 2002. Le vocabulaire de Levinas. Paris: Ellipses Éditions Marketing. 64p.

8. DALE, K. 2012. The Employee as 'Dish of the Day': the Ethics of Consuming/Consumed Self in Human Resource Management. J. Business Ethics. 111(1):13-24.
9. DÍAZ DE RADA, A. 2008. ¿Qué obstáculos encuentra la etnografía cuando se practica en las instituciones escolares? En: Jociles, M.I.; Franzé, A. (eds.) ¿Es la escuela el problema? Perspectivas Socioantropológicas de Etnografía y Educación. Madrid: Trotta. p.24-48.

10. FLAXER, D. 2011. Levinas in Practice: Mental Health Treatment in a Community-Based Clinic [sede web]. Seattle: Seattle University. Disponible desde internet en: http:/www.seattleu.edu/artsci/psychology/ default.aspx?id=7268 (Con acceso 01/04/2013).

11. GALLAGHER, S.; ZAHAVI, D. 2008. The Phenomenological Mind. An Introduction to Philosophy of Mind and Cognitive Sciences. London: Routledge. 244p.

12. GARZA, G.; LANDRUM, B. 2010. Ethics and the Primacy of the Other: A Levinasian foundation for Phenomenological Research. Indo-Pacific J. Phenomenol. 10(2):1-12.

13. GIERE, R. 2006. Scientific Perspectivism. Chicago: The University of Chicago Press. 160p.

14. GOODMAN, D.; BECKER, B. 2009. The psyche awakened: trauma, violence, and the other. Seattle: Seattle University. Disponible desde Internet en: http://www.seattleu.edu/artsci/psychology/default. aspx?id=7268 (Con acceso 01/04/2013).

15. HAACK, S. 2007. Defending Science -within reason. Between Scientism and Cynicism. New York: Prometheus Books. 411p.

16. HUSSERL, E. 2009. La filosofía, ciencia rigurosa. Madrid: Encuentro. 96p.

17. HUSSERL, E. 1986. Meditaciones Cartesianas. Madrid: Técnos. 246p.

18. HUSSERL, E. 1976. Die Krisis der europäischen Wissenschaften und die transzendentale Phänomenologie. Haag: Martinus Nijhoff. 555p.

19. IDARETA, F. 2012. Aproximación de la ética de Emmanuel Lévinas al trabajo social. Portularia. 12(1):1-8.

20. JARAMILLO, L.; AGUIRRE, J. 2010. Levinas y las ciencias sociales: fundamentos epistémicos desde la alteridad. Folios 31:3-20. 
21. JARAMILLO, L.; AGUIRRE, J. 2012. Una mirada al mundo ético de la vida a partir de la experiencia del dolor clínico. Rev. U.D.C.A. Act. \& Div. Cient. 15(1):9-17.

22. KUHN, T. 1996. The Structure of Scientific Revolutions. 3 ed. Chicago: The University of Chicago Press. 212p.

23. KUNZ, G. 1998. The paradox of power and weakness: Levinas and an alternative paradigm for Psychology. New York: State University of New York Press. 210p.

24. KUNZ, G. 2006. Interruptions: Levinas. J. Phenomenol. Psychol. 37(2):241-266.

25. KUNZ, G. 2007. An analysis of the psyche inspired by Emmanuel Levinas. Psychoanalytic Rev. 94(4):617638.

26. KUNZ, G. 2012. Conscience of a Conservative Psychologist: Return to the Mysteriously Illusive Psyche. Indo-Pacific J. Phenomenol. 12:1-13.

27. LATOUR, B.; WOOLGAR, S. 1979. Laboratory Life: The Social Construction of Scientific Facts. Princeton: Princeton University Press. 294p.

28. LE BRETON, D. 1999. Antropología del dolor. Barcelona: Seix Barral. 284p.

29. LEVINAS, E. 1967. La trace de l'autre. En: En Découvrant l'existence avec Husserl et Heidegger. Paris: Libraire Philosophique J. Vrin. p.187-202.

30. LEVINAS, E. 1990. Totalité et infini; essai sur l'extériorité. Paris: Le livre de Poche. 347p.

31. LEVINAS, E. 1991. Ética e Infinito. Madrid: Visor. 115p.

32. LEVINAS, E. 1993. La conciencia no intencional. En: Entre nosotros: ensayos para pensar en otro. Valencia: Pretextos. p.113-126.
33. LEVINAS, E. 2004. Autrement qu'être au-dela de l'essence. Paris: Le livre de Poche. 286p.

34. LYOTARD, J. 1979. La condition postmoderne: rapport sur le savoir. Paris: Minuit. 109p.

35. MACDONALD, H.; GOODMAN, D.; HOWE, K. 2011. The Ghetto Intern: Culture and Memory. Seattle: Seattle University. Disponible desde Internet en: http://www.seattleu.edu/artsci/psychology/default. aspx?id=7268 (con acceso 01/04/2013).

36. MÉLICH, J. 2010. Ética de la compasión. Barcelona: Herder. 320p.

37. ORANGE, D. 2011. Inclusion: Attitudes Toward Otherness For Clinical Therapeutics. Seattle: Seattle University. Disponible desde internet en: http://www.seattleu. edu/artsci/psychology/default.aspx?id =7268 (con acceso 01/04/2013).

38. SCHINDEL, E. 2013. En los zapatos del que sufre. Aproximaciones epistemológicas y éticas a los ex Centros Clandestinos de Detención. O ċcon qué calzado visitar un campo de concentración?" Papeles del CEIC, 201(93). Disponible desde Internet en: http:/www.identidadcolectiva.es/pdf/93.pdf (con acceso 01/04/2013).

39. SOKAL, A. 2010. Beyond the Hoax: Science, Philosophy and Culture. Oxford University Press (Reino Unido). 488p.

40. WALDENFELS, B. 2011. In place of the Other. Cont. Philos. 44:151-164.

41. ZAHAVI, D.; OVERGAARD, S. 2013. Intersubjectivity. En: LaFollette, $\mathrm{H}$. (ed.). The International Encyclopedia of Ethics. Oxford: Wiley Blackwell. p.1-8.

Recibido: Mayo 13 de 2013

Aceptado: Octubre 18 de 2013

Como citar:

Aguirre, J.C., Jaramillo, L.G. 2013. En el lugar del otro: posibilidades ético-epistemológicas para las ciencias humanas. Rev. U.D.C.A Act. \& Div. Cient. 16(2): 535-542. 